rostrum, it has other characters which would justify this action.

N. C. E. MHLER

Commonwealth Institute of Entomology, c/o British Museum (Natural History), London, S.W.7.

\section{Light Sensitivity of the Aquatic Flatworm Dendrocoelum lacteum}

WE have measured in various parts of the spectrum the amounts of radiation necessary to elicit in the planarian Dendrocoelum lacteum a characteristic response, namely, movement away from a source of light ${ }^{1}$. In each trial, one animal was dropped into a square 'Perspex' tank $(50 \mathrm{~cm} . \times 50 \mathrm{~cm}$., $10 \mathrm{~cm}$. deep) filled with lake water, in front of a calibrated source of spectral radiation placed outside the tank (opal electric bulb, 'neutral' wedges and colour filter $\left.{ }^{2}\right)$. D. lacteum, being milky-white in colour, could readily be detected with a red electric torch against the background of black paper placed under the tank. After $3 \mathrm{~min}$. the torch was switched on and it was seen whether the animal had moved at least $10 \mathrm{~cm}$. away from the light source in approximately the right direction. If so, this was taken as a positive response. The animal's movements were too slow for the observations to be appreciably affected by the light of the torch. In control experiments in which the colour filter was replaced by a piece of opaque cardboard, the number of animals giving a positive response was insignificant.

Trials were made with a number of animals using various spectral bands in the 'visible' range and a band around $0 \cdot 37 \mu$ in the near ultra-violet. For each spectral band, that intensity at which five out of the seventeen experimental animals responded was taken as the average threshold value. The individual thresholds varied considerably from one animal to another, and the accuracy which could be reached was markedly less than in the case of human vision. The sensitivity was a maximum in the neighbourhood of $0.5 \mu$; this seems in general agreement with the maximum near $0.53 \mu$ found by Viaud $^{3}$ in studies made, at higher intensities, of the orientation reactions of planarians having eyes.

The spectral sensitivity curve was found to be roughly similar to that of rod vision in the human aphakic eye-that is, an eye from which the lens has been removed. As the lens is responsible for the greater part of the light losses in the preretinal media of the human eye, the curve for $D$. lacteum must bear a general resemblance to the sensitivity curve of the human rod receptors. It should therefore also resemble the spectral absorption curve of the visual pigment rhodopsin; but it must be borne in mind that there is a discrepancy between the latter curve and the rod sensitivity curve at the violet end of the spectrum $^{5}$.

Relatively to the sensitivity to $0.51 \mu$, the sensitivity to radiation of about $0 \cdot 37 \mu$ was roughly a thousand times greater in D. lacteum than in the normal human eye with a lens. For this wavelength animals responded at an illumination at which they were quite invisible in the experimental tank to the dark-adapted, normal, human eye. The electric lamp itself, viewed through the $0 \cdot 37 \mu$ filter, was then barely visible - although it would, of course, have looked much brighter to an aphakic eye. In parts of the spectrum other than the near ultra-violet, violet and blue, the animals, when the illumination was high enough to elicit a positive response, could generally just be seen by the dark-adapted, normal, human eyo.

D. lacteum possesses two small but well-defined eyes, one on each side of the head. Taliaferro's experiments ${ }^{6}$ on other species of planarians showed that the reaction studied here was mediated through the eyes-while also suggesting that the individual sense-cells may possess a remarkable directional sensitivity. Assuming, then, that in the present case the reaction is mediated entirely through the eyes, and that the diameter of the effective pupil of the eye is a circle $0.08 \mathrm{~mm}$. in diameter, we calculate on the basis of absolute calibrations of the apparatus that five out of seventeen experimental animals responded in one series of experiments to an energy flux corresponding to about 40,000 quanta $(\lambda=$ $0.52 \mu$ ) per eye per sec., and in another series to about 4,000 quanta, or $15 \times 10^{-9}$ erg, per eye per sec.

In the case of man, the minimum flux of radiation which must enter the pupil of the eye for a small continuous light source to be seen is of the order of 750 quanta $(0.51 \mu)$, or $3 \times 10^{-8} \mathrm{erg}$, per sec. ${ }^{7}$. Thus the organisms studied here would have an absolute sensitivity close to that of the human eye. Artificial light-detectors of similar sensitivity (photo-multipliers with ancillary apparatus) might weigh about a million times as much as one specimen of $D$. lacteum. As stated, however, this discussion of absolute values rests on the assumption that the reaction is mediated entirely through the eye; experiments are in preparation to verify this assumption directly.

We are greatly indebted to $\mathrm{Mr}$. H. C. Gilson, director of the Windermere Laboratory of the Freshwater Biological Association, for facilities to carry out these experiments in his laboratory. The apparatus used was acquired through the support of the Nuffield Foundation and the Medical Research Council. One of us (F. H. C. M.) is the recipient of a. Nuffield Foundation Biological Scholarship.

M. H. Pirenne

F. H. C. MaRRIOTT

Physiology Department, Marischal College,

University of Aberdeen. Dec. 10.

${ }^{1}$ Ullyott, P., J. Exp. Biol., 13, 252 (1936).

See Denton, E. J., and Pirenne, M. H., J. Physiol., 123, 417 (1954); 125,181 (1954).

8 Viaud, G., "Année Biologique", 27, 365 (Paris, 1951).

- Wald, G., Science, 101, 653 (1945).

Stiles, W. S., Trans. Opt. Convent. Worshipful Company of Spectacle Makers, 97-107 (Spectacle Makers Company, London, 1948). - Taliaferro, W. H., J. Exp. Zool., 31, 59 (1920).

"Walsh, J. W. T., "Photometry" (2nd edit., London, 1953).

\section{An Asymmetrical Response of Teleost Melanophores}

Ballowitz ${ }^{1}$ Wyman ${ }^{2}$ and others have described several nerve fibres innervating the individual melanophores of Teleosts, and it is now generally accepted that these nerves are of autonomic origin. Whether the melanophore innervation is single, in volving aggregating fibres only, or double, including an antagonistic set of dispersing fibres, is still a matter of opinion (Parker ${ }^{3}$ ).

As a rule, section of a few melanophore nerve fibres in a white-adapted teleost fish causes an area of the related melanophores to disperse, the melanin granules being symmetrically distributed in the melanophore processes. Each of the surrounding 